\title{
Weighing Equipment Life Cycle Cost Based on Principal Component Analysis
}

\author{
Liang Xiong ${ }^{1,2}$, Huansong $\mathrm{Liu}^{1}$, Xin $\mathrm{Xu}^{1}$, and Weiwei Wang ${ }^{1,}$ \\ ${ }^{1}$ Military Economic Academy, Wuhan, 430035, China \\ ${ }^{2}$ Scocial Sciences Academy of Xinjiang, Urumqi, 830001, China
}

Keyword: Principal Component Analysis, Life Cycle Cost, Cost Tradeoff, Equipment.

\begin{abstract}
The equipment life cycle cost tradeoff based on principal component analysis can effectively defuse the split among its simplicity, comprehensiveness and integrity. Follow the steps to complete its tradeoff process in terms of primary data matrix construction, sample standardization, constructing indicator data matrix and correlation coefficient matrix, calculating characteristic vector and characteristic value, variance contribution rate calculation, and comprehensive evaluation.
\end{abstract}

\section{Introduction}

In the concept definition phase of the equipment, we tend to put forward several alternatives. With adequate performance indicators of all the alternatives, equipment life cycle cost tradeoff can be used to evaluate, analyze and compare life cycle costs of various alternatives, so as to ensure that the selective equipment is provided with lower life cycle costs with the ability to work in the same situation. As an important component of the equipment life cycle cost analysis, equipment life cycle cost tradeoff is a powerful technical support for decision making of all concerned unit.

In this paper, an attempt is made to build the model of equipment life cycle cost tradeoff analysis based on principal component analysis, which gives rise to a new way for equipment life cycle cost tradeoff.

\section{The feasibility of the introduction of principal component analysis in the equipment life cycle cost tradeoff}

Equipment life cycle cost tradeoff is involved with dozens of basic cost units in multiple aspects, such as equipment demonstration fee, development cost, original equipment cost, operating and support cost, and retirement and disposal cost. On condition that the equipment life cycle cost of each basic cost unit acts as the index that impact the equipment life cycle cost, then its breakdown structure of all cost units can be regarded as index system of equipment life cycle cost tradeoff. From this perspective, the process of equipment life cycle cost tradeoff analysis is essentially a kind of multi-index decision making and comprehensive evaluation process. However, in the equipment life cycle cost tradeoff analysis, index too much will increase the complexity of the tradeoff, and too little will have an influence on the comprehensive and integrity in tradeoff analysis. By the law of principal component analysis and with a series of transformation we can achieve our goals of "less variable numbers and more information acquired", thereby effectively defuse the split among simplicity, comprehensiveness and integrity of equipment life cycle cost tradeoff.

Mathematically, by principal component analysis using the method of dimension reduction, the multi-index is converted into a few of comprehensive index, which feedback most of the information in the original index system; And philosophically, principal component analysis in accordance with the thought of "key-point theory", concentrate on the important index of tradeoff quota system, in order to achieve clearly defined primary and secondary. From the basic thought and main characteristics of principal component analysis, it is observed that it is feasible that we introduce the principal component analysis to analyze equipment life cycle cost tradeoff. 


\section{Model building for equipment life cycle cost tradeoff based on principal component analysis}

\subsection{Constructing primary data matrix.}

Take each basic cost unit of the optional equipment life cycle cost breakdown structure as an tradeoff index of equipment life cycle cost. As for a device, there are $\mathrm{n}$ alternatives in the demonstration phase, and in its cost breakdown structure there are $\mathrm{p}$ basic cost units, which can be seen as $\mathrm{p}$ indicators reflecting the equipment life cycle cost. As a result, $\mathrm{p}$ indicators in each alternative are a sample value, a p-dimensions vector. $\mathrm{N}$ sets of data can be obtained from $\mathrm{n}$ alternatives, and each set of data has $p$ random variables, containing a total of $n \times p$ data. Thus a set of raw data matrix can be obtained.

$$
X=\left[\begin{array}{cccc}
x_{11} & x_{12} & \mathrm{~L} & x_{1 p} \\
x_{21} & x_{22} & \mathrm{~L} & x_{2 p} \\
\mathrm{M} & \mathrm{M} & \mathrm{M} & \mathrm{M} \\
x_{n 1} & x_{n 2} & \mathrm{~L} & x_{n p}
\end{array}\right] 。
$$

\subsection{Standardization of sample processing.}

Weighing equipment life cycle cost based on principal component analysis must first make data dimensionless. In general, the method of standardization for processing is adopted.

Firstly, calculate the mean and variance of each sample.

$$
\overline{x_{j}}=\frac{1}{n} \sum_{i=1}^{n} x_{i j} ; \quad S_{j}^{2}=\frac{1}{n-1} \sum_{i=1}^{n}\left(x_{i j}-\overline{x_{j}}\right)^{2}
$$

Among them, $\mathrm{j}=1,2, \ldots, \mathrm{p}$.

Secondly, standardize the sample. To standardize the sample, its purpose is to eliminate the dimensions and the difference among magnitude order of the variables, so as to satisfy standardization sample, which specific calculation procedure is as follows:

$$
x_{i j}{ }^{\prime}=\frac{x_{i j}-\overline{x_{j}}}{S_{j}}
$$

Among them, $i=1,2, \ldots, n ; j=1,2, \ldots, p$ 。

From the above result, the standardized raw data matrix can be obtained.

\subsection{Constructing correlation coefficient matrix of standardized index data matrix.}

Solve rij, bi-relation coefficient, of xij, each standardization indicator. As follows

$$
r_{i j}=\frac{1}{n-1} \sum_{k=1}^{n} x_{k i} x_{k j} \quad \mathrm{i}, \mathrm{j}=1,2, \ldots, \mathrm{p} \text { 。 }
$$

Among them, $\mathrm{i}, \mathrm{j}=1,2, \cdots, \mathrm{p}$ 。

The correlation coefficient matrix is constructed based on this, $R=\left\lfloor r_{i j}\right\rfloor_{p \times p}$ 。

\subsection{Calculating characteristic vector and characteristic value of the correlation matrix.}

By the characteristic equation, you can came at $\mathrm{p}$ characteristic roots: $\lambda \mathrm{g}(\mathrm{g}=1,2, \cdots, \mathrm{p})$ 。 These roots, in order of value, are $\lambda 1 \geqslant \lambda 2 \geqslant \cdots \lambda \mathrm{p} \geqslant 0$ 。 It is the variance of the principal component, the value of which describe the role played by the evaluation objects being described by each principal component. According to the characteristic equation, each characteristic value corresponds to a feature vector(lg1, lg2, $\cdots, \operatorname{lgp})$ 。

Turn the standardized indicator variable into principal component, as follows:

$$
F_{g}=l_{g 1} X_{1}+l_{g 2} X_{2}+\cdots+l_{g p} X_{p}
$$




\subsection{Calculating variance contribution rate and determining the numbers of principal components.}

Principal component analysis is to select a small number of m principal components $(\mathrm{m}<\mathrm{p})$ to make comprehensive evaluation, but also make the loss of information as little as possible. Choose $\mathrm{m}$ principal components, and principal components usually chosen in practice can make the cumulative contribution rate above $85 \%$, namely

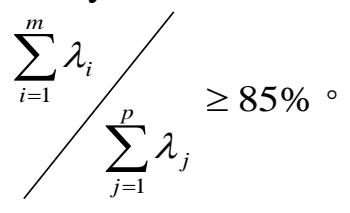

\subsection{Comprehensive evaluation to M principal components.}

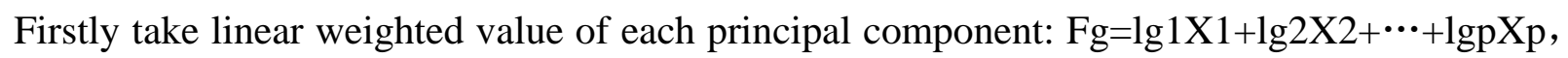
Then by weighted sum of m principal components, we acquire the final evaluation value.

$$
F=\sum_{g=1}^{m}\left(\sum_{g=1}^{\lambda_{g}} \lambda_{j}\right) F_{g} \text { 。 }
$$

After that, sort above result to arrive at the final tradeoff result.

\section{Empirical analysis}

In the concept phase of a device, the research activity puts forward three kinds of alternatives, numbered No.1, 2, and 3 respectively. These alternatives are roughly the same basic structure, and the determinated indicators of tactical and technical performance can meet the support requirement.

In this section, only select partial indicators of the alternatives to make empirical analysis. From the equipment life cycle cost breakdown structure we select a cost unit which consists of five basic cost unit, respectively as xi1,xi2, $\cdots$, xi5, among them, representation scheme serial number. The estimation results for cost unit of each scheme are as shown in table 1.

Table 1: the estimated results for a cost unit of a device

\begin{tabular}{llllll}
\hline & $x_{i 1}$ & $x_{i 2}$ & $x_{i 3}$ & $x_{i 4}$ & $x_{i 5}$ \\
\hline Scheme & 65 & 85 & 91 & 82 & 71 \\
Scheme & 80 & 69 & 79 & 83 & 83 \\
Scheme & 82 & 72 & 82 & 78 & 80 \\
\hline
\end{tabular}

Firstly, construct the primary data matrix. From table 1, we can get the primary data matrix, as follows:

$$
X=\left[\begin{array}{lllll}
65 & 85 & 91 & 82 & 71 \\
80 & 69 & 79 & 83 & 83 \\
82 & 72 & 82 & 78 & 80
\end{array}\right] \text { 。 }
$$

Secondly, standardize the original data matrix. After standardizing original data matrix, we can get:

$$
X^{\prime}=\left[\begin{array}{ccccc}
-1.1483 & 1.1370 & 1.1209 & 0.3779 & -1.1209 \\
0.4660 & -0.7443 & -0.8006 & 0.7559 & 0.8006 \\
0.6812 & -0.3915 & -0.3203 & -1.1338 & 0.3203
\end{array}\right]
$$

Thirdly, solve the correlation coefficient matrix of the standardized indicator data matrix, as follows: 


$$
R=\left[\begin{array}{ccccc}
1 & -0.9596 & -0.9392 & -0.4270 & 0.9392 \\
& 1 & 0.9979 & 0.1555 & -0.9979 \\
& & 1 & 0.0908 & -1 \\
& & & 1 & -0.0908 \\
& & & & 1
\end{array}\right]
$$

Fourthly, calculate the characteristic vector and characteristic value of the correlation matrices, as follows:

$$
\begin{gathered}
I=\left[\begin{array}{ccccc}
0.4919 & -0.1775 & 0.2458 & -0.0011 & 0.0000 \\
-0.4980 & -0.1022 & -0.3095 & 0.6169 & 0.0000 \\
-0.4936 & -0.1658 & -0.3179 & 0.1571 & -0.7071 \\
-0.1263 & 0.9519 & 0.1044 & -0.0408 & -0.0000 \\
0.5003 & 0.1565 & -0.8546 & 0.7701 & -0.7071
\end{array}\right] \\
\lambda=\left[\begin{array}{lllll}
3.9793 & 1.0322 & -0.0115 & -0.0001 & 0
\end{array}\right]
\end{gathered}
$$

Fifthly, calculate variance contribution rate and determine the numbers of principal components. Table 2 shows the characteristic value and variance contribution rate of each principal component. It is observed from the table that in the first two of these the characteristic root cumulative contribution rate are $100 \%$, which show that all the basic cost unit indicators of the cost unit have been basically included in the first two principal components, so you can use the first and the second principal component as comprehensive index analyzing and weighing the cost unit.

The characteristic vectors corresponding to the first two characteristic value are as follows respectively :

Then

$$
\left.\begin{array}{lllrl}
L 1=(0.4919 & -0.4980 & -0.4936 & -0.1263 & 0.5003
\end{array}\right)^{T}
$$

$$
\begin{aligned}
& F_{1}=0.4919 X_{1}-0.4950 X_{2}-0.4936 X_{3}-0.1263 X_{4}+0.5003 X_{5} \\
& F_{2}=-0.1775 X_{1}-0.1022 X_{2}-0.1658 X_{3}+0.9519 X_{4}+0.1565 X_{5}
\end{aligned}
$$

Comprehensive evaluation:

$\mathrm{F}=0.7959 \times \mathrm{F} 1+0.2064 \times \mathrm{F} 2$

Table 2: the characteristic value and variance contribution rate of each principal component

\begin{tabular}{cccc}
$\begin{array}{c}\text { Principal } \\
\text { Component }\end{array}$ & $\begin{array}{c}\text { characteristic } \\
\text { value }\end{array}$ & $\begin{array}{c}\text { variance } \\
\text { contribution } \\
\text { rate }(\%)\end{array}$ & $\begin{array}{c}\text { cumulative } \\
\text { contribution } \\
\text { rate }(\%)\end{array}$ \\
\hline 1 & 3.9793 & 79.59 & 79.59 \\
2 & 1.0322 & 20.64 & 100.000 \\
3 & -0.0115 & 0 & 100.000 \\
4 & -0.0001 & $-2 \mathrm{E}-15$ & 100.000 \\
5 & 0 & $-2.3 \mathrm{E}-15$ & 100.000 \\
\hline
\end{tabular}

Thus we can get comprehensive evaluation results of each alternative as shown in table 3.

Table 3: the comprehensive evaluation results of each alternative

\begin{tabular}{cccc}
\hline & $\mathrm{F}_{1}$ & $\mathrm{~F}_{2}$ & $\mathrm{~F}$ \\
\hline Scheme 1 & -30.1094 & 53.8550 & -12.9807 \\
Scheme 2 & -1.9786 & 57.2922 & 10.1126 \\
Scheme3 & -5.8228 & 51.2592 & 5.8103 \\
\hline
\end{tabular}

Thus the ranking for the life cycle cost of each scheme is scheme $2<$ scheme $3<$ scheme 1 . On the precondition of the performance of each scheme meeting the requirements, therefore, we should choose the lowest total cost, namely scheme 2 . 


\section{Summary}

Equipment life cycle cost tradeoff is an important component of the equipment life cycle cost analysis. Equipment life cycle cost tradeoff based on principal component analysis, from the ideas of dimensionality reduction and by the method of linear transformation, converting multi-indicators into a few of comprehensive indicators, with which feedback the most information of original quota system, has great theoretical and practical value.

At present, with all kinds of high and new technology applied in the equipment development and production in great quantities, as equipment has become increasingly complex, the basic cost units of its life cycle cost breakdown structure are increasingly more and more complicated. The method of equipment life cycle cost tradeoff based on principal component analysis has changed the limitations and subjectivity in the traditional weighing method, providing equipment life cycle cost tradeoff with a set of scientific, effective and practical procedure with comprehensive, reasonable and simple superiority, and will play an important role in the field of equipment management

\section{References}

[1]Hong Liu, Luo Junping, and Liu Jibing. Target Threat Assessment Based on Principal Component Analysis. Command and Control and Simulation, 2006 (2).

[2]Li Yanshuang, etc. The Application of Principal Component Analysis in the Multi-index Comprehensive Evaluation Method. Journal of Hebei University of Technology, 1999 (28).

[3]Liu.R.X,Kuang.J.andGong.Q. Principalcomponent regression analysis with spss, Computer Methods and Programs in Biomedicine. 2003(6) .

[4]Chen.Songcan,Zhu.Yulian. Subpattern-based principle component analysis , Pattern Recognition, 2004(5).

[5]Tang Chao. The Application of Principal Component Analysis in the Frontier Defence Intelligence Analysis. Information Exploration, 2008 (7).

[6]Gao Wenya, GeDan. Regional Industry Competitiveness Evaluation Based on Principal Component Analysis. Economic Forum. 2006 (02).

[7]Dou Jianbin, Lu Xinghua, Yang Pengyuan and Wang Qi. A Study on Evaluation of Equipment Combat Readiness Level Based on Improved Principal Component Analysis . Command and Control and Simulation, 2011 (01). 